\title{
Cooperative coverage-based lifetime prolongation for microgrid monitoring WSN in smart grid
}

\author{
Sujie Shao ${ }^{1 *}$, Lei Wu' ${ }^{1}$, Qinghang Zhang ${ }^{1}$, Neng Zhang ${ }^{1}$ and Kaixuan Wang ${ }^{2}$
}

*Correspondence:
buptssj@bupt.edu.cn
${ }^{1}$ State Key Laboratory
of Networking and Switching
Technology, Beijing
University of Posts
and Telecommunications,
Beijing, China
Full list of author information
is available at the end of the
article

*Correspondence: buptssj@bupt.edu.cn State Key Laboratory of Networking and Switching Technology, Beijing Beijing, China article

\begin{abstract}
To take full advantage of the flexibility of access and disconnection from smart grid, organizing distributed renewable energy resources in form of microgrid becomes one solution of energy replenishment in smart grid. A large amount of accurate and comprehensive information data are needed to be monitored by a variety of different types of sensors to guarantee the effective operation of this kind of microgrid. Energy consumption of microgrid monitoring WSN consequently becomes an issue. This paper presents a novel lifetime prolongation algorithm based on cooperative coverage of different types of sensors. Firstly, according to the requirements of monitoring business, the construction of cooperative coverage sets and connected monitoring WSN are discussed. Secondly, energy consumption is analyzed based on cooperative coverage. Finally, the cooperative coverage-based lifetime prolongation algorithm (CC-LP) is proposed. Both the energy consumption balancing inside the cooperative coverage set and the switching scheduling between cooperative coverage sets are discussed. Then, we draw into an improved ant colony optimization algorithm to calculate the switching scheduling. Simulation results show that this novel algorithm can effectively prolong the lifetime of monitoring WSN, especially in the monitoring area with a large deployed density of different types of sensors.
\end{abstract}

Keywords: Cooperative coverage, Lifetime prolongation, Energy consumption, Microgrid, Improved ant colony optimization

\section{Introduction}

Due to the growing consumption of energy and natural resources, distributed renewable energy resources gradually draw people's attention $[1,2]$. To take full advantage of the flexibility of access and disconnection from the power grid, organizing distributed renewable energy resources in form of microgrid as one solution of energy replenishment becomes a focusing issue in smart grid [3-5]. However, the inherent randomness and intermittence of energy supplement which are brought by the changes of status and environment of renewable energy resources may trouble microgrid operators to realize effective control and management of the distributed renewable energy resources, which may have an impact on stability of smart grid [6]. Therefore, it is necessary to monitor the devices, networks, resources and environment in the microgrid for scientific

(c) The Author(s) 2020. This article is licensed under a Creative Commons Attribution 4.0 International License, which permits use, sharing, adaptation, distribution and reproduction in any medium or format, as long as you give appropriate credit to the original author(s) and the source, provide a link to the Creative Commons licence, and indicate if changes were made. The images or other third party material in this article are included in the article's Creative Commons licence, unless indicated otherwise in a credit line to the material. If material is not included in the article's Creative Commons licence and your intended use is not permitted by statutory regulation or exceeds the permitted use, you will need to obtain permission directly from the copyright holder. To view a copy of this licence, visit http://creativecommons. org/licenses/by/4.0/. 
decision-making and efficient operation management. In order to obtain a large number of accurate and comprehensive information data about voltage, current, phase angle, temperature, humidity, frequency and others, a variety of different corresponding types of sensors need to be deployed [7-10]. At present, sensors are gradually integrated and miniaturized, and most of them are battery powered and their capacity of energy is limited.

For microgrid monitoring business, a variety types of data are required to work together to complete the relevant data analysis. The monitoring data like voltage, current, phase angle of some pivot points of microgrid should be analyzed together to get the information about power distribution and power loss for well managing and controlling the usage of renewable energy resources. In addition, temperature, humidity, frequency, smoke density and other environmental information data should be analyzed together to detect the probability and type of fault for quickly and effectively responding fault and well maintaining the microgrid. Different monitoring businesses are organized according to the requirements of different target points. Different sensors cooperate to be responsible for monitoring businesses. Therefore, the traditional coverage method can't well meet the different monitoring requirements of different target points in the microgrid. Moreover, energy consumption minimization and lifetime prolongation of the wireless sensor network (WSN) are another major problem when a huge and comprehensive data are required according to the different monitoring requirements.

To solve the problem, WSN for microgrid monitoring may not be organized according to the type of sensor again, but organized according to the type of monitoring business, which means all types of sensors involved by one single monitoring business should cooperate with each other to complete the monitoring business. These different types of sensors form a cooperative coverage set. The number of sensors in the cooperative coverage set is as small as possible, but only if the requirement of data collection has been achieved. The reduction of the number of sensors may destroy the connectivity of the network, but the coverage set can complete data forwarding through the cooperation between different types of sensors.

Obviously, more comprehensive raw data will be acquired for one single monitoring business based on cooperation of different types of sensors. So the corresponding decision-making of microgrid operation center may be more conveniently made, and effectiveness and efficiency of the monitoring business may consequently get great improvement. In addition, it means more potential choices of data forwarding for one single sensor and more reasonable communication process for monitoring WSN. Moreover, energy consumption control of monitoring WSN has more possibility for improvement.

Based on the above analysis about sensors cooperation in microgrid monitoring, we mainly study the following problems in this paper: (1) How to construct connected monitoring WSN based on cooperation of different types of sensors to meet the monitoring requirements in microgrid. (2) How to prolong the lifetime of WSN based on cooperation of different types of sensors.

To solve the two problems, the main points are the coverage of all monitoring target points and the energy consumption of sensors. A generalized reservation coverage scheduling algorithm is proposed in the literature [11]. It divides the whole WSN 
into several sensor sets, and each set can meet the general coverage requirement and work in turns to prolong the lifetime of WSN by scheduling these sets. Liu et al. [12] propose a quasi-grid-based cooperative coverage algorithm to reduce the number of active nodes and prolong the lifetime. Bao [13] prolongs the lifetime of WSNs by balancing the energy consumption inside the group of sensors and scheduling the working time among different groups of sensors. These literatures all aimed to reduce the number of sensors working at the same time while guaranteeing coverage, which can effectively prolong the lifetime of WSN, but they did not consider the cooperation of different types of sensors for multiple types of monitoring businesses. The k-coverage methods are analyzed in the literature [14, 15]; this deployment and working mechanism of sensors explain the redundant coverage of sensors for guaranteeing the quality of monitoring data and indicates more possibility of coverage and energy consumption scheduling for monitoring WSNs. Song et al. [16] propose a coverageaware unequal clustering protocol with load separation for ambient assisted living applications based on WSNs to achieve better performance and balance energy consumption for prolonging network lifetime. The above literatures mainly focus on the coverage performance of monitoring target while balancing energy consumption and ensuring the quality of data. However, the cooperation of different types of sensors is not considered. Xu et al. [17] widely discuss the energy consumption saving on the privacy-preserving data aggregation in WSNs by reducing communication overhead and energy expenditure of sensors. Li et al. [18] propose a data compression algorithm to enhance the lifetime of sensors in sea route monitoring system. Cao [19] divides working time of sensors into some short time periods and achieves lifetime prolongation by scheduling these working time periods. These works save the energy from the perspective of communication and data. They are very valuable to our work. Afshari et al. [20] propose a cooperative fault-tolerant control (CFTC) algorithm to address the problem of multiple actuator faults in autonomous AC microgrids. Two new distributed fault-tolerant control algorithms for the restoration of voltage and frequency in autonomous inverter-interfaced $\mathrm{AC}$ microgrids are proposed in the literature [21]. Dehkordi et al. [22] propose a novel distributed noise-resilient secondary control for voltage and frequency restoration of islanded microgrid inverter-based distributed generations (DGs) with an additive type of noise. Raeispour [23] proposes a distributed cooperative control protocol for inverter-based islanded microgrids. These works expand more application scenarios of the cooperation of sensors, including troubleshooting, distributed control, etc. Although all of the above research does not involve the cooperation of different types of sensors, the effective methods and ideas should be used as references.

Therefore, cooperation mechanism of different types of sensors for microgrid monitoring is introduced in this paper. The cooperative coverage set is firstly discussed to construct the connected monitoring WSN. Different types of sensors cooperate to form coverage sets. These coverage sets work in turns, that can reduce the number of sensors working at the same time while meeting the coverage rate. Secondly, cooperative coverage-based lifetime prolongation algorithm for microgrid monitoring WSN is proposed. Energy balance inside the cooperative coverage sets and switch scheduling between the cooperative coverage sets are included. Finally, in order to calculate the 
switch scheduling, we draw into an improved ant colony optimization algorithm. Our main contributions are as follows.

1 A cooperative coverage-based WSN for microgrid monitoring is proposed. In our model, sensors of one cooperative coverage set are simultaneously in work state to complete the monitoring business. We constructed cooperative coverage-based microgrid monitoring WSN to connecting a group of cooperative coverage sets that can combine to cover all the target points of the monitoring business with some communication sensors by applying the hierarchical clustering method. The number of sensors working at the same time keeps as small as possible to avoid wasting energy while ensuring data quality.

2 A cooperative coverage-based lifetime prolongation algorithm for microgrid monitoring WSN is proposed. What's more, we introduced an improved ant colony optimization algorithm to calculate the best cooperative coverage set switch sequence. Simulation results show that this novel algorithm can effectively prolong the lifetime of monitoring WSN with high time efficiency, especially in the monitoring area with large deployed density of different types of sensors. The numerical results verify the practicability and superiority of out algorithm, compared with several other policies.

The rest of this paper is organized as follows. In Sect. 2, cooperative coverage-based WSN for microgrid monitoring is introduced. In Sect. 3, cooperative coverage set of different types of sensors is studied. The cooperative coverage-based lifetime prolongation (CC-LP) algorithm for microgrid monitoring WSN is proposed in Sect. 4. Simulation results are analyzed in Sect. 5. Section 6 draws the conclusion.

\section{Methods}

In this section, the cooperative coverage-based WSN for distributed renewable energy resources oriented microgrid monitoring is discussed. According to the requirements of monitoring business, different types of sensors are organized. They cooperate with each other to complete the corresponding monitoring tasks.

\subsection{Network structure}

The distributed renewable energy resources oriented microgrid monitoring WSN based on cooperative coverage mainly involves charging stations, solar devices, wind turbines, energy storage devices, microgrid operation data center and different corresponding types of sensors. For the device status, microgrid operators utilize voltage, current and phase sensors to monitor the operating status and load information of these distributed power devices in real time. Meanwhile, different types of sensors are deployed for specific devices, for example, wind speed sensor and the direction sensor are used to evaluate the operating status of the wind turbine; light sensor and the temperature sensor are deployed to collect light and temperature data around the solar station. For environmental information, smoke sensors, temperature and humidity sensors and others need to be deployed to obtain a large number of accurate and comprehensive information data. In general, these sensors are mainly used to monitor the status information of the relevant 
devices and environmental information, such as voltage, current, phase angle, temperature, humidity, frequency and so on.

As shown in Fig. 1, different types of sensors are deployed to monitoring the devices in the microgrid to complete the monitoring business according to the corresponding requirements. Each type of sensor covers a fixed size monitoring area which is expressed by the corresponding circle with different types of dotted line.

The deployment of sensors needs to consider not only the Euclidean distance between the device and the sensor, but also the electrical topology of the device. If there are bifurcations on electrical wires, the phase in every branch needs to be acquired. In order to ensure comprehensiveness of the collected data and fault tolerance in the monitoring network, we adopted a redundant deployment scheme. In this scheme, redundant sensors guarantee that the phase of each branch can be monitored based on the electrical topology. Monitoring data can be sent to the access point and data center by data cooperative communications among different types of sensors.

Each sensor covers at least one monitoring target point. Each monitoring target point may be covered by at least one sensor because of the existence of different types of sensors. It is feasible to select part of sensors in the monitoring area to complete the monitoring business. Thus, it is unnecessary for every sensor to be in work state, which indicates the possibility of pursuing energy consumption minimization and lifetime prolongation while the monitoring business is ensured.

\subsection{Cooperative coverage}

In the microgrid monitoring WSN, cooperative coverage of varied types of sensors mainly contains two meanings. First one is cooperative coverage with regard to monitoring target points, which is the cooperation between sensors with the same type. We call it the first type of cooperative coverage. The other one is cooperative coverage with

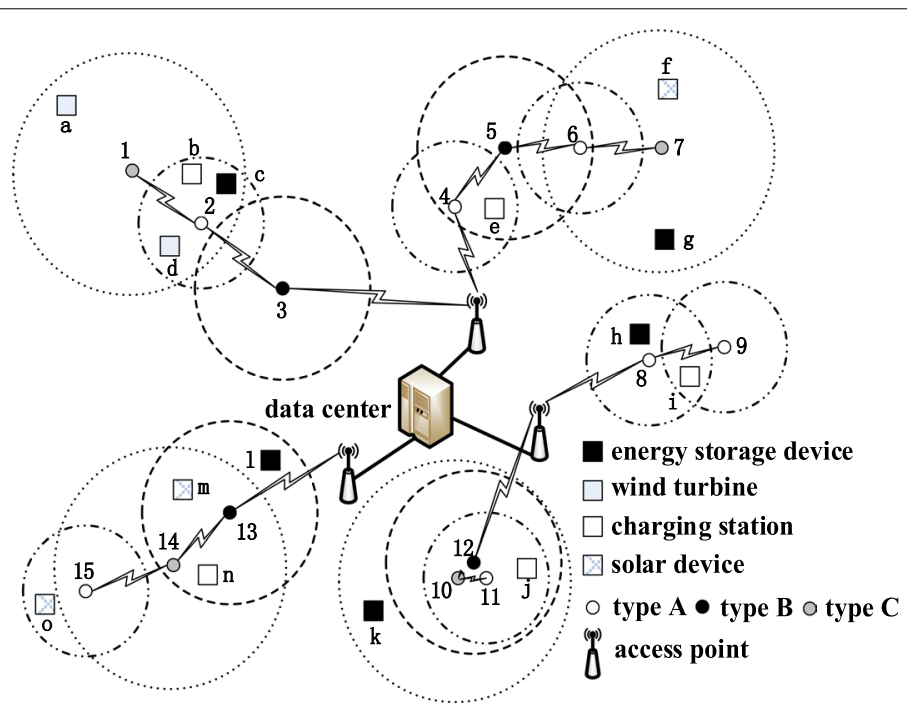

Fig. 1 Cooperative coverage-based WSN for microgrid monitoring. This figure shows the network structure of the monitoring microgrid; different types of sensors are deployed to monitoring the devices in the microgrid to complete the monitoring business 
regard to data communication, which is the cooperation between sensors with the different types. We call it the second type of cooperative coverage.

In order to guarantee the data integrity of monitoring business, at least one sensor is needed to be deployed for each monitoring target point to monitor its data change in principle. Each single sensor has a clear monitoring coverage range. If one monitoring target point is covered by at least two sensors with the same type at the same time, activating one of these sensors is theoretically enough to complete the data acquisition of this monitoring target point. Thus, sensors with the same type can cooperate together and select sensors as few as possible to complete the monitoring tasks according to monitoring requirements, which we call the first type of cooperative coverage.

We denote $r\left(s_{i}, p_{j}\right)$ as the distance between sensor $s_{i}$ and monitoring target point $p_{j}$. Generally, the signal strength of sensor decreases as the $r\left(s_{i}, p_{j}\right)$ increases. To guarantee the quality of monitoring data, we denote $r_{0}$ as the threshold of $r\left(s_{i}, p_{j}\right)$, which means monitoring data of sensor $s_{i}$ about target point $p_{j}$ is valid if $r\left(s_{i}, p_{j}\right) \leq r_{0}$. For instance, monitoring target point $i$ (a charging station) is covered by two sensors with the same type 8 and 9 at the same time in Fig. 1. Then, sensors 8 and 9 can cooperate with each other. From the perspective of monitoring task of target point $i$, only one of the two sensors needs to be in work state.

The first type of cooperative coverage will obviously reduce the number of single type of sensors that needs to be activated. However, it may destroy the connectivity of the initial deployment network, so that parts of sensors may be in an isolated state. It is necessary to select some other types of sensors to complete the data forwarding based on the first type of cooperative coverage. Thus, the second type of cooperative coverage is needed.

If different types of sensors can cooperate and communicate with each other as long as they are within their communication range. Then, it is unnecessary to consider the connectivity and activate other sensors with the same type to complete the data forwarding during the decision-making process of the first type of cooperative coverage. Once there is another type of sensor selected according to the first type of cooperative coverage within the communication range, the data forwarding can be completed. Therefore, the connectivity and communication of monitoring WSN are completed via the cooperation of different types of sensors, which we call the second type of cooperative coverage.

The number of activated sensors of whole monitoring WSN is reduced, resulting in saving unnecessary consumed energy of sensors. For instance, sensor 2 can forward data of sensor 1 to the data center, and it is not necessary to activate other type $\mathrm{C}$ sensors as long as sensor 2 has enough energy. Obviously, sensor 2 must be activated according to the requirements of monitoring business. The number of activated sensors is reduced.

With the two types of cooperative coverage, sensors which are in the working state can be divided into three categories: sensors that only undertake communication tasks, sensors that only undertake monitoring tasks and sensors that undertake both communication and monitoring tasks. We call them communication sensor, monitoring sensor and dual-function sensor, respectively. In Fig. 1, sensors 2, 4, 5, 8, 10, 12, 13 and 14 are the dual-function sensors. Sensors 1, 7, 9, 11 and 15 are the monitoring sensors. Sensors 3 and 6 are the communication sensors. Obviously, communication sensors only play the role of connecting the other sensors to form a connected monitoring WSN. 
The monitoring data are sensed by the monitoring sensors and dual-function sensors, both of them can cover all the monitoring target points. The three types of sensor roles may be mutually transformed over time because the sensor selection will change as the energy consumption of sensors change over time.

\subsection{Energy consumption of single sensor}

The working sensor needs to monitor the status of target point and communicate with other sensors in WSN. Since energy consumption of sensing data is much smaller than energy consumption of communications, only energy consumption of communications is considered in this paper. Energy consumption of communications can be divided into energy consumption of receiving data and energy consumption of transmitting data. According to the first-order wireless communication energy consumption model, we calculate the energy consumption of receiving one monitoring data and energy consumption of transmitting one monitoring data as $e_{t}=e_{\mathrm{rd}}+e_{\mathrm{amp}}, e_{r}=e_{\mathrm{rd}}$, respectively. $e_{\mathrm{rd}}$ is energy consumed by radio devices, $e_{\mathrm{amp}}$ is energy consumed by power amplifier, which is related to the communication distance between two sensors. For receiving data and transmitting data, $e_{\mathrm{rd}}$ is same.

We denote $k$ and $k_{0}$ as the number of monitoring data received by one single sensor and the number of monitoring data sensed by one single sensor during one time period, respectively. Thus, from the perspective of cooperative coverage, energy consumption of dual-function sensor $s_{i}$ in time period $\mathrm{t}$ is calculated as

$$
\begin{aligned}
e_{i, t} & =k \times e_{r}+\left(k+k_{0}\right) \times e_{t}=k \times e_{\mathrm{rd}}+\left(k+k_{0}\right) \times\left(e_{\mathrm{rd}}+e_{\mathrm{amp}}\right) \\
& =\left(2 k+k_{0}\right) \times e_{r d}+\left(k+k_{0}\right) \times e_{\mathrm{amp}} .
\end{aligned}
$$

Similarly, energy consumption of communication sensor and that of monitoring sensor in time period $\mathrm{t}$ are, respectively, calculated as

$$
\begin{aligned}
& e_{i, t}=k \cdot e_{r}+k \cdot e_{t}=2 k \cdot e_{\mathrm{rd}}+k \cdot e_{\mathrm{amp}} \\
& e_{i, t}=k_{0} \cdot e_{t}=k_{0} \cdot e_{\mathrm{rd}}+k_{0} \cdot e_{\mathrm{amp}} .
\end{aligned}
$$

Then, we can calculate energy consumption of sensors, and select appropriate sensors to be activated and organized for the lifetime prolongation of monitoring WSN.

\subsection{Communication technologies}

In the cooperative coverage-based microgrid monitoring WSN, sensors communicate among themselves and the access points. The access points communicate with the remote data processing and control center which store monitoring data and send control messages. We mainly use ZigBee technology to enable communication among sensors, as it is widely used in low-power networks. In addition, most types of sensors, which are available in smart grid monitoring market, use ZigBee for communication. ZigBee and other short-range radio technologies are supported by sensors communicating with access points. The access points send monitoring data to the remote data processing and control center through WLAN, wireless cellular network or high-speed wired network 
technologies. Similarly, control messages are forwarded to the corresponding sensors by access points.

Due to the changing energy consumption of different roles of sensors and the different business requirements, we need to select appropriate sensors to construct connected microgrid monitoring WSN, so that the effective cooperative coverage can be actually realized. In the next section, the cooperative coverage set is discussed, and we adopt it as the basic element to construct the connected microgrid monitoring WSN based on cooperative coverage.

\section{Construction of cooperative coverage-based monitoring WSN}

In this section, the cooperative coverage set is discussed. Sensors of one cooperative coverage set are simultaneously in work state to complete the monitoring business. Then, cooperative coverage-based microgrid monitoring WSN is constructed by connecting a group of cooperative coverage sets that can combine to cover all the target points of the monitoring business with some communication sensors.

\subsection{Coverage cooperative set}

At a specific moment, the cooperative coverage-based monitoring WSN will be split into several disconnected groups if we remove all communication sensors, and each group can be called a cooperative coverage set. The composition of each cooperative coverage set and number of the cooperative coverage sets depend on the monitoring requirements and the initial deployment distribution of sensors in the corresponding microgrid monitoring area. For instance, sensors 1 and 2, sensors 4 and 5, sensors 10, 11 and 12, sensors 13,14 and 15 compose a cooperative coverage set, respectively.

We denote $\mathrm{MB}\left(m, \mathrm{TP}_{1}, \mathrm{TP}_{2}, \ldots, \mathrm{TP}_{m}\right)$ as the monitoring business, where $m$ is the number of types of sensors involved by the monitoring business, and $\mathrm{TP}_{i}$ is the set of monitoring target points of type $i$ sensors. All target points in $\mathrm{TP}_{i}$ should be covered by the combination of type $i$ sensors in all cooperative coverage sets.

We denote $s_{i}(j, \mathrm{TP})$ as the single sensor $s_{i}$, where $j(j=1,2, \ldots, m)$ is the type $\mathrm{ID}, i$ is the sensor ID, and TP is the set of target points within the coverage range of $s_{i}$. We denote $\mathrm{CCS}_{l}\left(\mathrm{SS}, \mathrm{TP}_{1}, \mathrm{TP}_{2}, \ldots, \mathrm{TP}_{m}\right)$ as the cooperative coverage set, where $l$ is the cooperative coverage set ID, $\mathrm{SS}$ is the set of its sensors, and $\mathrm{TP}_{i}$ is the set of monitoring target points of type $i$ sensors within the combined coverage range of sensors in SS.

The method of constructing a cooperative coverage set is as follows. If $s_{i}$ can communicate with one sensor of $\mathrm{CCS}_{l}$ and $s_{i} \cdot \mathrm{TP} \not \subset \mathrm{CCS}_{l} \cdot \mathrm{TP}_{j}$, then $s_{i}$ can join $\mathrm{CCS}_{l}$ and become one sensor member of $\mathrm{CCS}_{l}$. And $\mathrm{CCS}_{l}$ is calculated as

$$
\left\{\begin{array}{l}
\mathrm{CCS}_{l} \cdot \mathrm{SS}=\mathrm{CCS}_{l} \cdot \mathrm{SS} \cup\left\{s_{i}(j, \mathrm{TP})\right\} \\
\mathrm{CCS}_{l} \cdot \mathrm{TP}_{j}=s_{i} \cdot \mathrm{TP} \cup \mathrm{CCS}_{l} \cdot \mathrm{TP}_{j}
\end{array} .\right.
$$

We keep repeating this process to construct the cooperative coverage set until no sensor can join $\mathrm{CCS}_{l}$. For two different types of sensors $s_{a}$ and $s_{b}$, if they can communicate with each other, then they can compose a cooperative coverage set. For two same type of sensors $s_{a}$ and $s_{b}$, if they can communicate with each other and $s_{a} \cdot \mathrm{TP} \not \subset s_{b} \cdot \mathrm{TP} \& \& s_{b} \cdot \mathrm{TP} \not \subset s_{a} \cdot \mathrm{TP}$, then they can compose a cooperative coverage set. 
With the above method, a group of cooperative coverage sets of monitoring business $\mathrm{MB}\left(m, \mathrm{TP}_{1}, \mathrm{TP}_{2}, \ldots, \mathrm{TP}_{m}\right)$ would be constructed.

Because sensor joins the cooperative coverage set one by one, there may be a phenomenon that all target points of one sensor may be covered by other same type of sensors in the cooperative coverage set. If the cooperative coverage set is still connected after removing this sensor, then we call this sensor a redundant sensor of this cooperative coverage set. We call the cooperative coverage set without any redundant sensor a minimum cooperative coverage set. Generally, we need to construct the minimum cooperative coverage set for saving energy.

\subsection{Connected monitoring WSN construction}

The cooperative coverage set can meet the data monitoring requirements of all target points with the sensors as few as possible, but these sensors may not be connected. The monitoring data may not be forwarded to the remote microgrid data center. Therefore, we need to further select some communication sensors to construct a connected monitoring WSN based on cooperative coverage. The hierarchical clustering method is adopted.

All sensors in a cooperative coverage set are connected; thus, we can consider the cooperative coverage set as an entire communication group. The distance of two communication groups is denoted as the shortest one among the distances from sensor in one group to sensor in the other group. The two communication groups with the shortest distance are selected after calculating all distances of any two groups. Then, sensors that can connect the two communication groups are selected, while making sure that the number of these sensors is as few as possible. Then, these two communication groups and the selected communication sensors are merged into a new communication group.

In the process of merging communication groups, it is important to ensure that the number of communication sensors in the selected communication group is the minimum. Then, we calculate the shortest distance by network hop counts and build the set of candidate paths with the least hops. After that, if the boundary of a communication group is obvious, a remote node will be chosen, otherwise we choose the communication node with the largest residual energy. In the process of node communication, if there are multiple paths and the residual energy of the current communication node is enough, the subgroups will be adjusted according to the energy consumption. If the residual energy of the current communication node is insufficient, the communication node will be changed dynamically according to the residual energy.

The above process is repeated until a group of cooperative coverage sets of monitoring business $\mathrm{MB}\left(m, \mathrm{TP}_{1}, \mathrm{TP}_{2}, \ldots, \mathrm{TP}_{m}\right)$ are all connected. Then, the construction of connected monitoring WSN has been finished. The hierarchical clustering-based construction method of connected monitoring WSN is shown in Fig. 2.

\subsection{Energy consumption of monitoring WSN}

Sensors in a cooperative coverage set are in work state at the same time. If one sensor fails due to energy exhaustion, the corresponding area would become a monitoring blind area, resulting in failure of the entire cooperative coverage set. There is a Barrel Effect for lifetime of cooperative coverage set. Therefore, energy consumption is another 


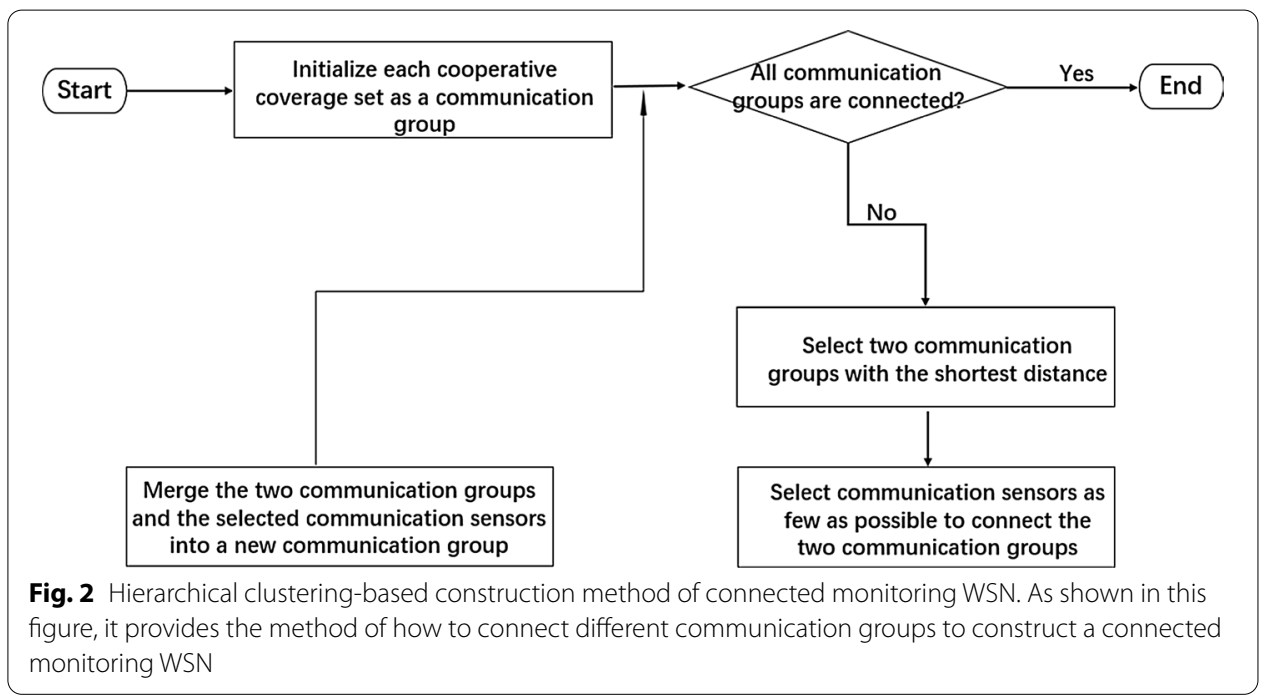

important factor except monitoring coverage and connectivity when we construct the cooperative coverage set.

We assume that the number of monitoring data sensed by one single sensor during one time period is a fixed value. Then, the total number of monitoring data sensed by the cooperative coverage set during one time period is known. We assume that the communication route within the cooperative coverage set does not change during one time period. Thus, energy consumption of each sensor during one time period can be calculated.

We denote $k_{i, t}$ as the number of data received by $s_{i}$ during time period $t$, which is the sum of data forwarded by all the descendants of $s_{i}$ in the cooperative coverage set during time period $t$. Then, energy consumption of dual-function sensor $s_{i}$ in time period $t$ is calculated as

$$
\begin{aligned}
e_{i, t} & =k_{i, t} \cdot e_{r}+\left(k_{i, t}+k_{0}\right) \cdot e_{t} \\
& =k_{i, t} \cdot e_{\mathrm{rd}}+\left(k_{i, t}+k_{0}\right) \cdot\left(e_{\mathrm{rd}}+e_{\mathrm{amp}}\right) \\
& =k_{i, t} \cdot\left(2 e_{\mathrm{rd}}+e_{\mathrm{amp}}\right)+k_{0} \cdot\left(e_{\mathrm{rd}}+e_{\mathrm{amp}}\right) .
\end{aligned}
$$

The energy consumption of monitoring sensor is still calculated according to Formula (3).

We call the sensor that forward data to communication sensor out of the cooperative coverage set the head sensor. According to Formula (5), energy consumption of head sensor is the most. Dual-function sensors near the head sensor have the relatively more energy consumption. Energy consumption of the monitoring sensor is the least. Thus, energy consumption of sensors in cooperative coverage set is closely related to the communication route of sensors. The more times data are forwarded, the more energy is consumed by the cooperative coverage set.

To balance the energy consumption, communication route inside the cooperative coverage set needs to be adjusted over time, which mainly involves the head sensor. If the energy of the whole cooperative coverage set can't support the monitoring tasks, another new cooperative coverage set need to be constructed to replace the current one, which 
may be called cooperative coverage set switching. Both of the two ways may change the selection of communication sensors. The specific methods of adjusting communication route and switching cooperative coverage set are discussed in the next section.

Energy consumption of communication sensor during one time period would keep constant if the cooperative coverage set that it connected and the direction of data forwarding keep unchanged. According to Formula (2), it can be easily calculated. Then, the energy consumption of the current monitoring WSN can be calculated.

To play the greatest advantage of cooperative coverage set, we need to prolong its working time as much as possible and further prolong the lifetime of whole microgrid monitoring WSN based on cooperative coverage according to the actual energy consumption. We focus on this issue in the next section.

\section{Cooperative coverage-based lifetime prolongation algorithm}

In this section, the CC-LP algorithm is proposed. Both the energy consumption balancing inside the cooperative coverage set and the switching scheduling between cooperative coverage sets are discussed. Then, we draw into an improved ant colony optimization algorithm to calculate the switching scheduling.

\subsection{Energy consumption balancing inside the cooperative coverage set}

Cooperative coverage set reduces the number of sensors that is simultaneously in work state and makes good use of the redundant deployment of the different types of sensors, but the tasks of some key sensors may be inevitably increased, which may lead to extra energy consumption of these sensors to the disadvantage of the continuous work of the cooperative coverage set. Thus, balancing the energy consumption of sensors inside the cooperative coverage set is needed.

We denote $E_{i, t}$ as the residual energy of $s_{i}$ at the beginning of time period $t$. There are $m$ sensors in the cooperative coverage set $\mathrm{CCS}_{l}$. Therefore, the number of time periods $n_{l}$ that $\mathrm{CCS}_{l}$ can continuously run is calculated as

$$
n_{l}=\min _{i=\{1,2, \cdots, m\}}\left(\left\lfloor E_{i, t} / e_{i, t}\right\rfloor\right) .
$$

According to Formulas (3) and (5), head sensor and its neighbor sensors may be the energy bottleneck of $\mathrm{CCS}_{l}$, which depend on the communication route within $\mathrm{CCS}_{l}$. Thus, to eliminate the energy bottleneck, we need to adjust the communication route.

For adjusting communication route, there are main two ways. One is fixing the head sensor and adjusting communication path from other sensors to the head sensor. The other is changing the head sensor and optimizing the consequent communication route. The latter one may need to activate other communication sensors to guarantee the connectivity of WSN. Here, we only discuss the changing without activating other communication sensors. If there are several sensors that can communicate with the current communication sensor, the one with the most residual energy among them should be selected as the head sensor at the beginning of each time period.

Changing a new head sensor lead to the network topology in the cooperative coverage set being rebuilt. According to the ZigBee technology, extra routing update messages need to be sent to rebuild the network, resulting in additional energy consumption. 
However, considering the position and composition of sensors in the cooperative coverage set, the head sensor may be not changed if there are no alternative sensors. Even if the head sensor changes, on the one hand, the head sensor was changed only in the cooperative coverage set; thus, messages are mainly delivered by sensors inside the set and there is no other communication sensor to be activated. On the other hand, the number of sensors in one cooperative coverage set and routing update messages is relatively small, so the extra energy consumption of delivering routing update messages is less than the energy consumption of transmitting monitoring data, and has a weak effect on the overall performance of our algorithm.

For a given head sensor, the communication route optimization can be transformed to how to find the maximum value of minimum residual energy $E_{i, t+w}$ after $w$ time periods that $\mathrm{CCS}_{l}$ continuously run. The problem can be expressed as Formula (7):

$$
E=\max \left(\min _{i=1,2, \ldots, m\}}\left(E_{i, t+w}\right)\right), \quad w \in\left[1, n_{l}\right]
$$

where $E$ is the energy bottleneck of $\mathrm{CCS}_{l}$.

After one time period, the residual energy of $s_{i}$ can be calculated as

$$
E_{i, t+1}=E_{i, t}-e_{i, t} .
$$

Then, $E_{i, t+w}$ will be calculated by repeating Formula (8) $w$ times, and the energy consumption balancing inside the cooperative coverage set is achieved.

\subsection{Switching scheduling between cooperative coverage sets}

Moreover, when a cooperative coverage set fails due to the energy exhaustion or it needs to stop working due to the switching scheduling for lifetime prolongation, a new cooperative coverage set is needed to be constructed to complete the monitoring tasks instead. Then, some sensors may need to be activated from the sleep state and some other sensors need to sleep again, which inevitably lead to extra energy consumption of these sensors too. Frequent switching between different cooperative coverage sets may not necessarily enable the lifetime prolongation of microgrid monitoring WSN. Therefore, selecting appropriate cooperative coverage set and switching at the appropriate time are also needed.

There may be more than one cooperative coverage set that can complete the monitoring tasks. For reducing the switching times, we sort all these cooperative coverage sets according to their minimum residual energy of sensor in descending order. Then, we select the switching candidate cooperative coverage set in order.

Moreover, one sensor may be selected by different cooperative coverage sets at different time periods. Then, its energy consumption of the previous switching round definitely affects the working time of the next switching round. Thus, we set another energy threshold $p$ to determine the appropriate switching timing of current cooperative coverage set in some cases, which means Formula (7) may be constrained. We denote $E_{i}^{c}$ as the energy capacity of $s_{i}$. If $E_{i, t} \leq p E_{i}^{c}$, we can consider that $s_{i}$ is no longer suitable for continuous working. When more than half of sensors happen like this, we can consider that the current cooperative coverage set is no longer suitable for continuous working if there is another cooperative coverage set that can be switched. However, this constraint 
is not necessary because our switching scheduling can still continue if all of the cooperative coverage sets meet the condition, which obviously means the lifetime of the monitoring WSN will end soon.

To simplify the problem, we still use $\mathrm{CCS}_{l}$ to denote the switching cooperative coverage set. Then, the number of time periods $n_{l}$ that $\mathrm{CCS}_{l}$ can continuously run is calculated as Formula (9):

$$
n_{l}=\min \left(n_{l, 0}, n_{l, 1}\right)
$$

where

$$
n_{l, 0}=\min _{i=\{1,2, \ldots, m-c\}}\left(\left\lfloor E_{i, t} / e_{i, t}\right\rfloor\right),
$$

and

$$
n_{l, 1}=\min _{i=\{m-c+1, m-c+2, \ldots, m\}}\left(\left\lfloor\left(E_{i, t}-E_{0}\right) / e_{i, t}\right\rfloor\right) .
$$

$E_{0}$ is denoted as the energy consumption of activating a sensor, $m$ and $c$ are total number of sensors in $\mathrm{CCS}_{l}$ and number of sensors that are activated to construct $\mathrm{CCS}_{l}$, respectively.

If $n_{l}=0, \mathrm{CCS}_{l}$ definitely can't be the switching candidate cooperative coverage set. Thus, we can select number from $\left[1, n_{l}\right]$ to schedule the work state or sleep state of $\mathrm{CCS}_{l}$.

Assuming that lifetime of monitoring WSN ends after $q$ times switching of cooperative coverage set, the problem of lifetime prolongation of monitoring WSN is transformed into how to select the appropriate number of working time periods of cooperative coverage sets to prolong the lifetime. The problem can be expressed as Formula (12), where $s n$ is the maximum lifetime of monitoring $\mathrm{WSN}, w_{j}$ is the number of time periods that $\mathrm{CCS}_{l}$ continuously run in the $j$-th switching.

$$
s n=\max \left(\sum_{j=1}^{q} w_{j}\right), \quad w_{j} \in\left[1, n_{l}\right] .
$$

For each $w_{j}$, it derived from Formula (7), and $E_{i, t},(i=m-c+1, m-c+1, \ldots, m)$ in Formula (8) is modified as $E_{i, t}-E_{0} . m$ and $c$ are the specific ones in the $j$-th cooperative coverage set.

\subsection{CC-LP algorithm based on improved ant colony optimization}

In this subsection, the CC-LP algorithm based on improved ant colony optimization is proposed to calculate the best cooperative coverage set switch sequence. Compared with the common ant colony optimization algorithm, we have made some improvements in our algorithm. The improvement schemes of our algorithm are as follows.

1 To select the next cooperative coverage set, we propose a probability formula based on pheromone, residual energy and switch energy consumption of coverage set.

2 If we use the common ACO algorithm, the same cooperative coverage set will not be repeatedly selected. But in our model, when the cooperative coverage set has sufficient energy, the same cooperative coverage set should be repeatedly selected to 
avoid frequent switching and excessive energy consumption. Therefore, we optimized the pheromone update method of our improved ant colony optimization algorithm, expanded the selectable path, and searched for more solution space.

We denote the number of ants as $M$ and the number of cooperative coverage sets as n. Each ant has a tabu table that records the cooperative coverage sets whose residual energy is not enough, these cooperative coverage sets cannot be selected. Firstly, we randomly place all ants on cooperative coverage sets, and assume the pheromone in coverage set is $\tau_{i j}(0)\left(\tau_{i j}(0)\right.$ is a constant). Secondly, we calculate the probability that ant $k$ switches from $\mathrm{CCS}_{i}$ to $\mathrm{CCS}_{j}$ based on Formula (13):

$$
P_{i j}(t)=\left\{\begin{array}{ll}
\frac{\left[\tau_{i j}(t)\right]^{\alpha} *\left[L_{j}\right]^{\beta} *\left[\eta_{i j}\right]^{\gamma}}{\sum_{s \in \mathrm{Tabu}_{k}}\left[\tau_{i s}(t)\right]^{\alpha} *\left[L_{s}\right]^{\beta} *\left[\eta_{i s}\right]^{\gamma}}, & j \notin \mathrm{Tabu}_{k} \\
0, & j \in \mathrm{Tabu}_{k}
\end{array} .\right.
$$

In Formula (13), $k$ is the ID of ants and $\mathrm{Tabu}_{k}$ is the tabu table of ant $k$. We denote $\tau_{i j}$ as the pheromone of $\mathrm{CCS}_{i}$ toCCS $j$. Furthermore, $L_{j}$ is the residual energy, and Formula (12) describes the calculation of $L_{j}$ :

$$
L_{j}=\sum_{i=\{1,2, \cdots, m\}}\left(\left\lfloor E_{i, t} / e_{i, t}\right\rfloor\right) / m+\min \left(\left\lfloor E_{i, t} / e_{i, t}\right\rfloor\right) .
$$

In Formula (14), $L_{j}$ obtained by adding the average and minimum values of the remaining time periods of all nodes in cooperative coverage set $j$.

We denote $\eta_{i j}$ as the heuristic information of $\mathrm{CCS}_{i}$ to $\mathrm{CCS}_{j}$. Formula (13) describes the calculation of $\eta_{i j}$ :

$$
\eta_{i j}=\frac{1}{\sum_{s \in \mathrm{ccs}_{j}} E_{a c t}(s), s \notin \mathrm{ccs}_{i}} .
$$

In Formula (15), we denote $E_{\text {act }}(s)$ as the additional energy consumption of node $s$. If node $s$ in $\mathrm{CCS}_{j}$ not be included in $\mathrm{CCS}_{i}$, additional energy consumption $E_{\text {act }}(s)$ will be calculated in this formula.

What's more, in Formula (13), $\alpha$ is a heuristic factor, which reflects the relative importance of pheromone. The larger $\alpha$ is, the more likely the ants are to select the previous path, and the less the randomness of the ant colony search. $\beta$ and $\gamma$, respectively, reflect the relative importance of residual energy and switch energy consumption when an ant selects next cooperative coverage set. The larger $\beta$ and $\gamma$ are, the more likely the ants fall into the local optimum.

These three parameters are very important parameters in the algorithm, and the selection method will affect global convergence and calculating efficiency of the ant colony algorithm. Meanwhile, functions of the parameters in the ant colony algorithm are closely related. If these parameters are not configured properly, the solution speed will be very slow and the lifetime of network will be dissatisfied.

After all ants select the coverage set through Formula (13) and run to the end, we only perform global pheromone update on the best path. On the one hand, it allows the ants to find a better path based on the residual concentration of pheromone on the path. On 
the other hand, it allows the ants to search for optimal solution at a faster speed and promotes the convergence of the algorithm. In our model, in order to maximize the lifetime of a network, the network may select $\mathrm{CCS}_{i}$ until the energy consumption of $\mathrm{CCS}_{i}$ reaches the threshold. Formulas (16) and (17) describe the update method of global pheromone:

$$
\begin{aligned}
& \Delta \tau_{i j}(t)= \begin{cases}Q / L_{\text {best }}, & i \neq j \\
Q /\left(L_{\text {best }} * C_{i}\right) & i=j\end{cases} \\
& \tau_{i j}(t+1)=(1-\rho) \tau_{i j}(t)+\mathrm{RHQ} * \Delta \tau_{i j}(t) .
\end{aligned}
$$

In Formulas (16) and (17), we denote $Q$ as the sum of pheromone for an ant. $L_{\text {best }}$ is the length of the best path and RHQ is the attenuation rate of pheromone. $\rho$ represents the pheromone volatilization rate, and $\rho$ has an impact on the search ability and convergence speed of our algorithm. We denote $C_{i}$ as the number of select the same $\mathrm{CCS}_{i}$ continuously. Obviously, as $C_{i}$ increases, the average energy of $\mathrm{CCS}_{i}$ will gradually decrease. When an ant selects the same $\mathrm{CCS}_{i}$ in the next iteration, $\mathrm{C}_{i}$ ensures that the incremental value of pheromone is inversely proportional to the number of select times. Thus, the energy of sensors in the monitoring network can be balanced and the lifetime of monitoring network can be prolonged.

In our algorithm, the number of iteration $N$ is set. In each iteration, there will be $\mathrm{M}$ ants searching the switching path according to Formula (13) at the same time. The search process of each any is the switching sequence of the coverage sets until it cannot find a set of sensors that can meet the requirements of monitoring business. When the search process ends, the lifetime of monitoring WSN also ends. The complexity of the algorithm is related to the number of cooperative coverage sets constructed. However, it is hard to obtain an accurate function mapping of the number of sensors and the number of corresponding cooperative coverage sets, because the location and number of sensors in the monitoring WSN are random and network model is complicated. We assume the number of constructed coverage sets is C, then the complexity of CC-LP-IACO is $O=\left(N \times C^{2} \times M\right)$. The algorithm is shown in Algorithm 1. 


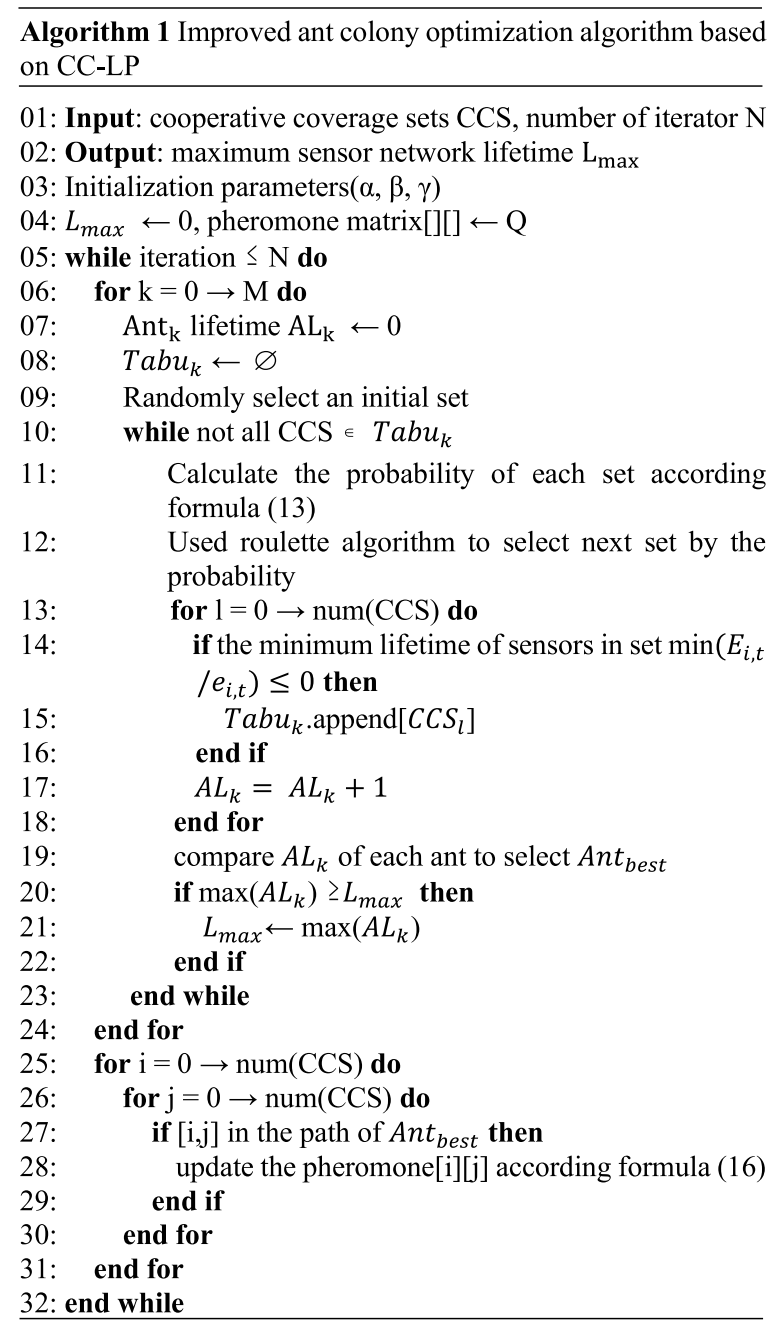

\section{Simulation results and discussion}

In this section, the performance of our proposed CC-LP algorithm is evaluated. There are 3 types of sensors in the simulated monitoring WSN. Their coverage ranges are $10 \mathrm{~m}$, $15 \mathrm{~m}$ and $20 \mathrm{~m}$, respectively. The number of each type of sensors is same. Each type of sensor needs to monitor 10 target points that are randomly scattered in $100 * 100 \mathrm{~m}^{2}$ area. There is one access point in the center of the area. Our simulation was programmed by python3.6 and was run on the computer with i5-7300HQ CPU @ $2.50 \mathrm{GHz}$ and 8 GB of RAM.

In this paper, energy consumption of node sending and receiving data is the same as the energy consumption model of wireless sensor network in the literature [24], which is a first-order wireless communication model. Each resource node generates a certain number of data packets in each time period. The size of the data packet is 100 Byte, and the initial energy of the sensor node is $0.05 \mathrm{~J}$. The parameter settings are shown in Table 1. 
Table 1 Parameter settings

\begin{tabular}{lll}
\hline Parameter & Values & Unit \\
\hline Node distribution region & $100 \times 100$ & - \\
Position of the base station & $(50,50)$ & - \\
The number of nodes & $60,80,100,120,150$ & J \\
The initial energy of each node & 0.05 & bit \\
Total size of data & 800 & bit \\
$e_{r d}$ & $5 \times 10^{-8} \mathrm{~J}$ & bit \\
$e_{\text {amp }}$ & $1 \times 10^{-11} \mathrm{~J}$
\end{tabular}

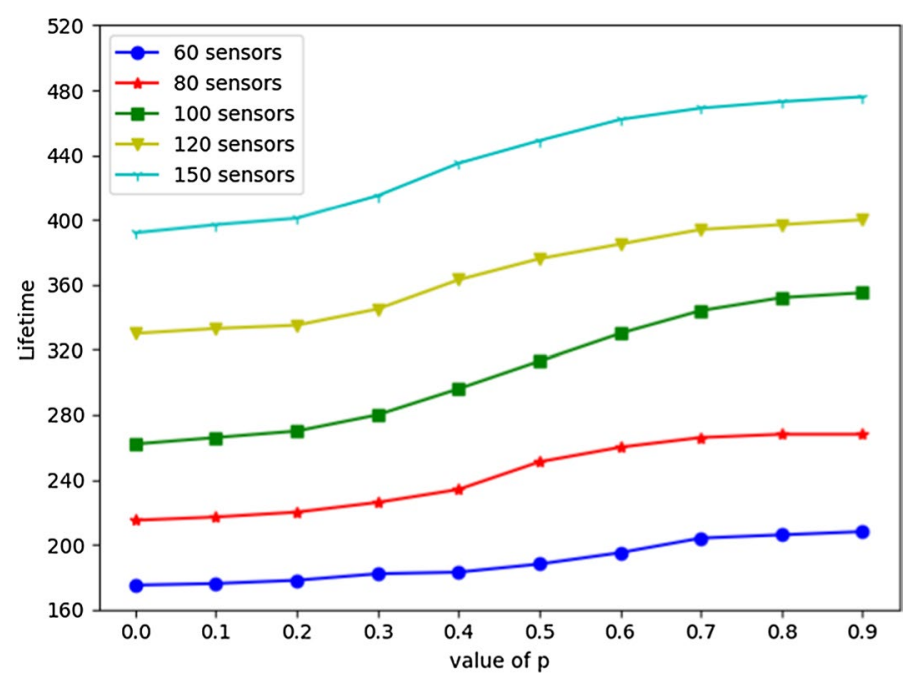

Fig. 3 Lifetime of monitoring WSN with the change of $p$ for five sensor densities. This figure shows the lifetime of monitoring WSN from the value of $p$ varies for five different sensor densities

In the design of our CC-LP, the value of $p$ is the factor that will actually affect the performance of lifetime prolongation. Firstly, 60, 80, 100,120 and 150 sensors are deployed in the area, and simulations are carried out under these four sensor densities to determine the optimal value of $p$. The number of sensors in real microgrid is much greater than this number, but the mechanism of cooperative coverage of real microgrid is the same as our simulation. Secondly, in order to optimize the CC-LP algorithm, we compare the influence of $\alpha, \beta$ and $\gamma$ to the network and the performance of different numbers of nodes. Thirdly, to further evaluate performance of fault detection, we compare CC-LP with the greedy algorithm and LP-based heuristic proposed in the literature [18]. The greedy algorithm switches the cooperative coverage that can meet the requirement of monitor business and own the most residual energy, regardless of the switch consumption to the next switch round. LP-based heuristic selects nodes to be added to the coverage set by transforming the selection process to integer programming while spending a time period. To ensure statistical validity, the data used in the simulation results analysis are averaged and all simulation experiments are repeated 100 times.

Figures 3 and 4, respectively, show the lifetime of monitoring WSN and number of cooperative coverage set. 


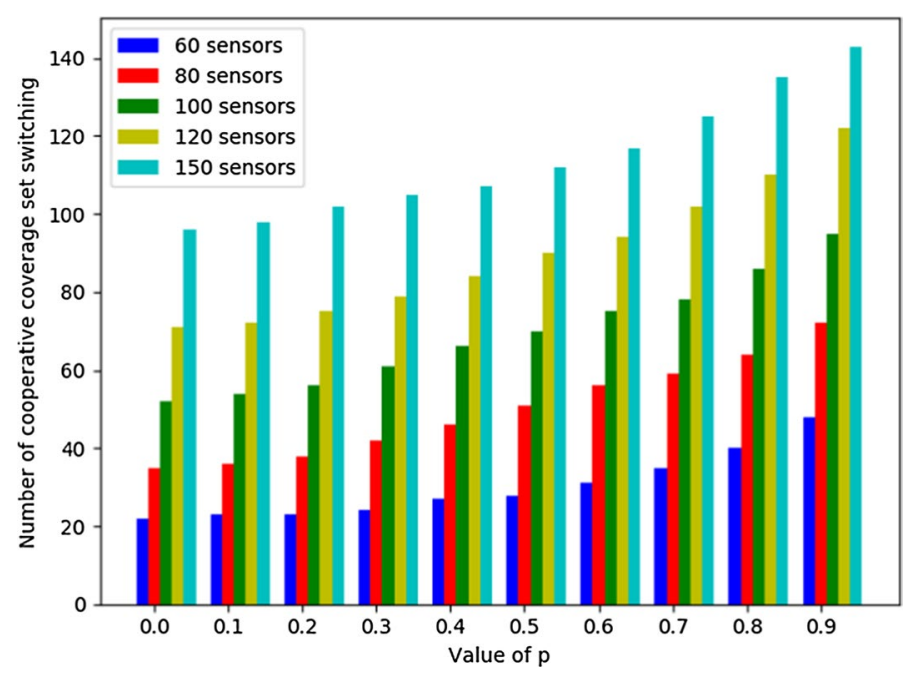

Fig. 4 Number of cooperative coverage set switching with the change of $p$ for five sensor densities. This figure shows the number of cooperative coverage set switching from the value of $p$ varies for five different sensor densities

switching with the increasing value of $p$ and five different sensor densities. Figure 3 shows that the lifetime of monitoring WSN increases as the value of $p$ increases. The larger the value of $p$ is, the more frequent switching operations of cooperative coverage sets happen, which results in more optimized energy consumption of sensors and more working time periods of whole monitoring WSN. The threshold p determines the switch timing of the coverage set selected in the last switch. When the energy of the half of sensors in the coverage set is less than $p E_{i}^{c}$, the coverage set that is currently working must be switched. While the value of $\mathrm{p}$ is too small, the energy of some key sensor nodes in the coverage set will be consumed early, so that the monitoring business cannot be completed. Although there are many other sensor nodes in WSN, and the energy of these sensors is still rich, they cannot form a cooperative coverage set to meet the requirements of the monitoring business. While the value of $p$ is larger, the energy consumption of each coverage concentrated can be better uniformed, thereby extending the lifetime of the entire monitoring WSN. However, in some cases, the larger value of $p$ does not necessarily mean the better performance of CC-LP algorithm because the activating and sleeping operations of sensor result in extra energy consumption of sensor. As shown in Figs. 3 and 4, number of cooperative coverage set switching keeps rapidly increasing but lifetime of monitoring WSN keeps slowly increasing as $p$ increases and $p \geq 0.7$. Moreover, the frequent switching is not conducive to the stability of the monitoring system. Therefore, we consider 0.7 as the optimized value of $p$ in our CC-LP algorithm.

Figure 5 shows the influence of three parameters $\alpha, \beta$ and $\gamma$ to the lifetime of network. Based on literature $[25,26]$, we select a number of appropriate parameter values. These three parameters can effectively affect the final convergence result of our algorithm. The larger the value of alpha, beta and gamma, the greater the amount of calculation and the longer the calculation time in our simulation. Moreover, it is the relative value of these three parameters that mainly affects the performance of 


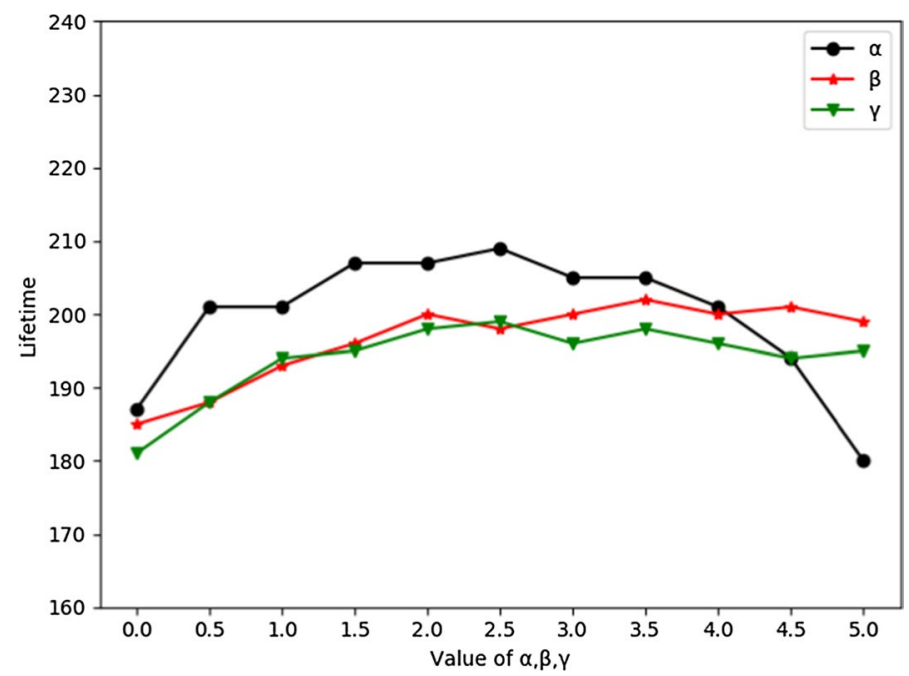

Fig. 5 Lifetime of monitoring WSN with the change of $a, \beta, \gamma$. This figure depicts the influence of three parameters $\alpha, \beta$ and $\gamma$ to the lifetime of the monitoring network

the algorithm. We choose the range of $\alpha, \beta$ and $\gamma$ is $[0-5.0]$ and their interval is 0.5 . Figure 5 depicts that when $\alpha$ is too small or $\alpha$ is too large, the performance of CC-LP algorithm will be dissatisfied. When $\alpha$ is too large, the algorithm completely relies on the guidance of pheromone to search, which leads to the strong positive feedback and premature convergence. When $\alpha$ is too small, the algorithm relies too much on the energy and switching cost of the cooperative coverage set. It is difficult to find the optimal solution by continuously selecting the cover set with better energy and switching cost at local time. Therefore, when $\alpha \in[1.3,3.5]$, the performance of CC-LP algorithm is satisfactory and the lifetime of monitoring WSN is longer.

Meanwhile, Fig. 5 also depicts that $\beta, \gamma \in[2.0,4.0]$ is satisfactory. Because when $\beta$ and $\gamma$ are too small, ant colony falls into random search and the lifetime of network will not increase with the number of cooperative coverage set switch. And when $\beta$ and $\gamma$ are too large, although the lifetime is satisfactory, the convergence performance will be not good.

Then, we combine the value of $\mathrm{p}$ and the three different parameters $\alpha, \beta, \gamma$ to further analyze the impact of $p$ and $\alpha, \beta, \gamma$ on the lifetime of monitoring WSN. Figure 6 further validates the data of Fig. 3, the lifetime of monitoring WSN increases as the value of $\mathrm{p}$ increases, but when the value of $p>0.7$, the increase in the lifetime is limited, and the number of cooperative coverage switching increases rapidly. Besides, Fig. 6 shows that when $\alpha, \beta, \gamma=2.0$ at the same time, the lifetime of monitoring WSN is significantly higher than when one of them is equal to 5.0. This indicates that if the weight of a factor is too large, the convergence performance of the colony becomes worse, and an optimal solution cannot be obtained. What's more, when $\alpha$ is equal to 5.0, the lifetime of monitoring WSN is obviously lower than when the weight of one of $\beta, \gamma$ is too large, which is in line with the trend shown in Fig. 5.

In the end, we compare the performance of our CC-LP algorithm with the greedy algorithm and the LP-based heuristic algorithm. Figure 7 shows the lifetime of 


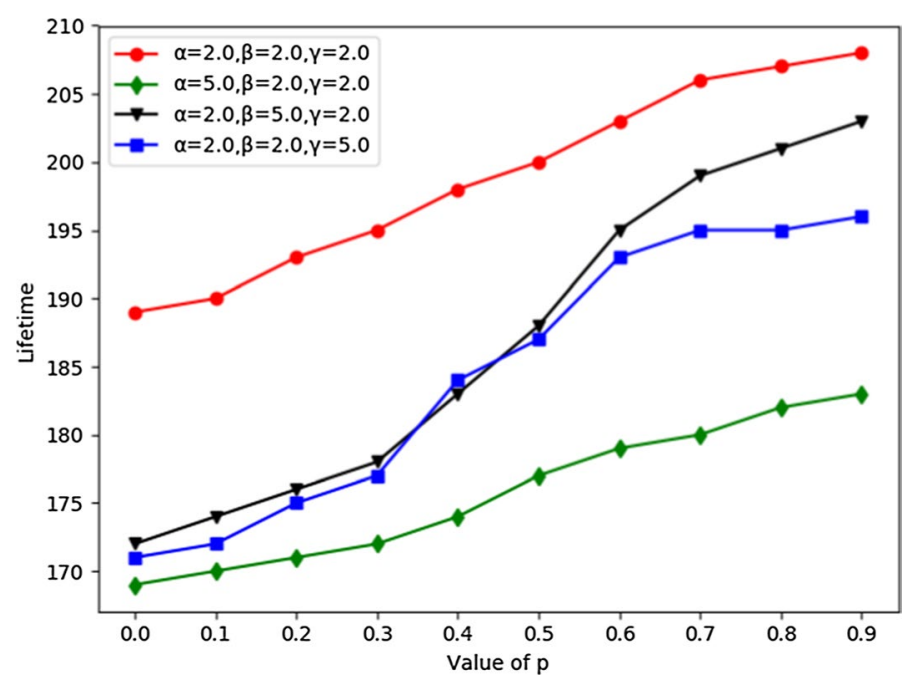

Fig. 6 Lifetime of monitoring WSN with the change of the combination of $p$ and $a, \beta, \gamma$. This figure shows the lifetime of monitoring WSN with the change of $\mathrm{p}$ for four different combinations of parameters of $a, \beta, \gamma$

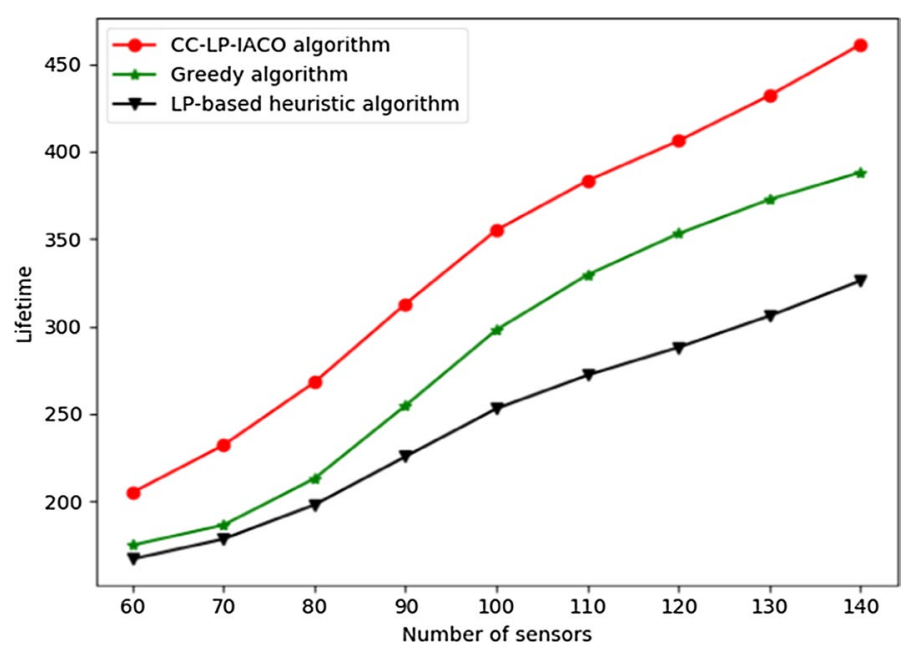

Fig. 7 Lifetime of monitoring WSN for three algorithms. This figure depicts the comparison of lifetime of monitoring WSN for three algorithms, greedy, LP based, CC-LP-IACO with $p=0.7, a=2, \beta=3, \gamma=3$

monitoring WSN in $p=0.7, \alpha=2, \beta=3, \gamma=3$. As shown in Fig. 7, lifetime of monitoring WSN of CC-LP is the longest.

The CC-LP algorithm can effectively balance the energy of sensors in the WSN, avoiding the excessive energy consumption of some key sensors, and thus prolonging the lifetime of monitoring WSN. The other two algorithms consume the energy of some sensors without considering the impact on the working time of next switch round, so that it cannot form a connected network. The greedy algorithm selects the cooperative coverage set with the highest average energy in every time slot, resulting in frequent switching of cooperative coverage sets. The sensors in sleep state will be activated frequently, thereby increasing energy consumption. As the number of 
sensors increases, CC-LP algorithm has a $15-20 \%$ improvement over greedy algorithm at each value of sensor density. And the differences of performance keep increasing because the selection of cooperative coverage sets can be more flexible in greater scope and energy of each sensor can be more effectively used.

Figure 8 shows that the number of cooperative coverage set switching of CC-LP is also the least when $p=0.7, \alpha=2, \beta=3, \gamma=3$, and it does not rapidly increase as the number of sensors increases. Thus, simulation results show that our CC-LP algorithm can effectively prolong the lifetime of monitoring WSN with high time efficiency, especially in the monitoring area with large deployed density of different types of sensors.

In this paper, it is assumed that the sensors are scattered in the regular area. Actually, the monitoring area of WSN is irregular in microgrid. However, efficiency of cooperative coverage is figured out, and simulation results show that our CC-LP can be used as a reference in microgrid monitoring WSN.

\section{Conclusion}

Energy consumption is one of the important issues of the distributed renewable energy resources oriented microgrid monitoring WSN in smart grid. This paper presents a novel cooperative coverage-based lifetime prolongation algorithm (CC-LP) for microgrid monitoring WSN. We describe the working mechanism of cooperative coverage of different types of sensors in detail. According to the requirements of monitoring business, constructions of the cooperative coverage sets and the connected monitoring WSN are discussed, respectively. Based on cooperative coverage, we analyze energy consumption of single sensor of monitoring WSN. We discuss the energy consumption balancing inside the cooperative coverage set and the switching scheduling between cooperative coverage sets, and then propose the CC-LP algorithm based on improved ant colony optimization to the switching sequence of cooperative coverage set. In the simulation, we adjusted various values of parameters to get better convergence performance of our

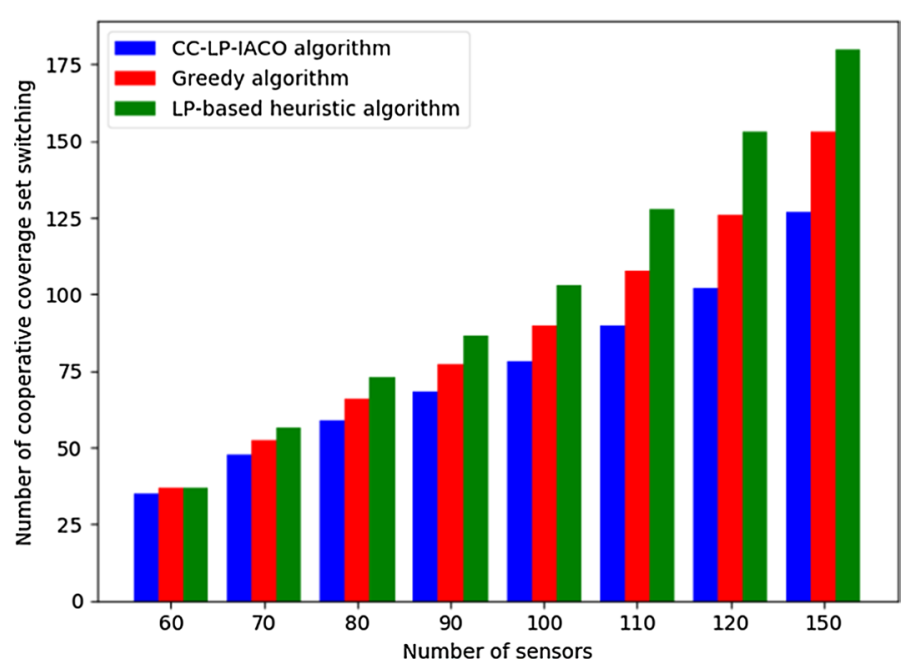

Fig. 8 Number of cooperative coverage set switching for three algorithms. This figure describes the comparison of number of cooperative coverage set switching for three algorithms, greedy, LP based, CC-LP-IACO with $p=0.7, a=2, \beta=3, \gamma=3$ 
algorithm. Compared with two other algorithms, simulation results show that this novel algorithm can effectively prolong the lifetime of monitoring WSN with high time efficiency, especially in the monitoring area with large deployed density of different types of sensors.

\section{Abbreviations}

WSN: Wireless sensor network; DG: Distributed generation; WLAN: Wireless local area network; ACO: Ant colony optimization; LP: Linear programming.

\section{Acknowledgements}

This work is supported by Test bed construction of industrial Internet platform in specific scenes (new mode), the Beijing Natural Science Foundation through the Research on Adaptive Fault Recovery Mechanism for Electric Power loT under Grant 4194085, the Fundamental Research Funds for the Central Universities under Grant 2019RC08, the National Natural Science Foundation of China (61672108) and the Natural Science Foundation of Shanxi Province, China, under Grant No. 201801D121116.

\section{Authors' contributions}

SJS proposed the main idea; he is the main writer of this paper. LW and QHZ performed the simulation and analyzed the result. NZ gave some important suggestions for the paper and revised the paper; KXW revised the paper. All authors read and approved the final manuscript.

\section{Availability of data and materials}

The datasets used and analyzed during the current study are available from the corresponding author on reasonable request.

\section{Competing interests}

The authors declare no conflict of interest.

\section{Author details}

1 State Key Laboratory of Networking and Switching Technology, Beijing University of Posts and Telecommunications, Beijing, China. ${ }^{2}$ Department of Information, Shanxi University of Finance and Economics, Taiyuan, China.

Received: 13 July 2020 Accepted: 6 November 2020

Published online: 07 December 2020

\section{References}

1. H.E. Brown, S. Suryanarayanna, S.A. Natarajan, S. Rajopadhye, Improving reliability of islanded distribution systems with distributed renewable energy resources. IEEE Trans. Smart Grid. 3(4), 2028-2038 (2012)

2. S. Lakshminarayana, T.Q.S. Quek, P.H. Vincet, Cooperation and storage tradeoffs in power grids with renewable energy resources. IEEE J. Sel. Areas Commun. 32(7), 1386-1397 (2014)

3. S.M. Hakimi, S.M. Moghaddastafreshi, Optimal planning of a smart microgrid including demand response and intermittent renewable energy resources. IEEE Trans. Smart Grid 5(6), 2889-2900 (2014)

4. W. Su, J. Wang, J. Roh, Stochastic energy scheduling in microgrids with intermittent renewable energy resources. IEEE Trans. Smart Grid 5(4), 1876-1883 (2014)

5. R.Q. Wang, Research on multi-objective optimization design and coordinated control of distributed generation and microgrid. Ph.D. Thesis, Shandong University, Jinan, China, 16 April 2013

6. J. Zhang, L. Su, Y. Chen, J. Su, L. Wnag, Energy management of microgrid and its control strategy. Power Syst. Technol. 35, 23-29 (2011)

7. X. Wang, Q.L. Liang, Efficient sensor selection schemes for wireless sensor networks in microgrid. IEEE Syst. J. 12(1), 539-547 (2018)

8. H. Chen, B.Q. Wei, D.S. Ma, Energy storage and management system with carbon nanotube supercapacitor and multidirectional power delivery capability for autonomous wireless sensor nodes. IEEE Trans. Power Electron. 25(12), 2897-2909 (2010)

9. M. Tariq, P.H. Vincent, Real time electricity theft detection in microgrids through wireless sensor networks, in Proceedings of the 2016 15th IEEE Sensors Conference, Orlando, FL, 30 October-2 November 2016

10. L.M. Andreoni, J. Francisco, G.M. Marcelo, Implementation of wireless remote monitoring and control of solar photovoltaic (PV) system, in Proceedings of the 2012 IEEE/PES Transmission and Distribution: Latin America Conference and Exposition, Montevideo, URUGUAY, 3-5 September 2012

11. X.F. Liu, J.N. Cao, S.J. Tang, P. Quo, A generalized coverage-preserving scheduling in WSNs: a case study in structural health monitoring, in Proceedings of the IEEE Conference on Computer Communications, Toronto, CANADA, 27 April-2 May 2014

12. Z. Liu, Z.D. Qiu, Quasi-grid based cooperative coverage algorithm for wireless sensor networks. J. Electron. Inf. Technol. 32(10), 2531-2535 (2012)

13. X. Bao, Research on lifetime prolonging algorithms for wireless sensor networks. Ph.D. Thesis, Chang'an University, Xi'an, China, 2 November 2011 
14. X.H. Xu, M. Song, Restricted coverage in wireless networks, in Proceedings of the IEEE Conference on Computer Communications, Toronto, Canada, 27 April-2 May 2014

15. H.M. Ammari, S.K. Das, Centralized and clustered k-coverage protocols for wireless sensor networks. IEEE Trans. Comput. 61(1), 118-133 (2012)

16. X.Y. Song, T. Wen, W. Sun, D.Q. Zhang, Q. Guo, Q.L. Zhang, A coverage-aware unequal clustering protocol with load separation for Ambient Assisted Living based on wireless sensor networks. China Commun. 13(5), 47-55 (2016)

17. J. Xu, G. Yang, Z.Y. Chen, Q.Q. Wang, A survey on the privacy-preserving data aggregation in wireless sensor networks. China Commun. 12(5), 162-180 (2015)

18. Y. Li, Z.S. Zhang, H.F. Wei, X.M. Chai, X.P. Zhu, H.L. Zhu, Sea route monitoring system using wireless sensor network based on the data compression algorithm. China Commun. 11(13), 179-186 (2014)

19. X. Cao, Research on extending lifetime of surveillance systems of wireless sensor networks. M.S. Thesis, Nanjing University, Nanjing, China, May 2013

20. A. Afshari, M. Karrari, H.R. Baghaee, G.B. Gharehpetian, S. Karrari, Cooperative fault-tolerant control of microgrids under switching communication topology. IEEE Trans. Smart Grid 11(3), 1866-1879 (2020)

21. A. Afshari, M.N. Karrari, H.R. Baghaee, G.B. Gharehpetian, Distributed fault-tolerant voltage/frequency synchronization in autonomous AC microgrids. IEEE Trans. Power Syst. 35(5), 3774-3789 (2020)

22. N.M. Dehkordi, H.R. Baghaee, N. Sadati, J.M. Guerrero, Distributed noise-resilient secondary voltage and frequency control for islanded microgrids. IEEE Trans. Smart Grid. 10(4), 3780-3790 (2019)

23. M. Raeispour, H. Atrianfar, H.R. Baghaee, G.B. Gharehpetian, Distributed LMI-based control of heterogeneous microgrids considering fixed time-delays and switching topologies. IET Renew. Power Gener. 10, 1-11 (2020)

24. W.B. Heinzelman, A.P. Chandrakasan, H. Balakrishnan, Application-specific protocol architectures for wireless networks. IEEE Trans. Wirel. Commun. 1(4), 660-670 (2002)

25. M. Dorigo, V. Maniezzo, A. Colorai, Ant system: optimization by a colony of cooperating agents. IEEE Trans. Syst. Man Cybern. Part B 26(1), 29-41 (1996)

26. S.C. Zhan, J. Xu, J. Wu, The optimal selection on the parameters of the ant colony algorithm. Bull. Sci. Technol. 5, 381-386 (2003)

\section{Publisher's Note}

Springer Nature remains neutral with regard to jurisdictional claims in published maps and institutional affiliations.

\section{Submit your manuscript to a SpringerOpen ${ }^{\circ}$ journal and benefit from:}

- Convenient online submission

Rigorous peer review

- Open access: articles freely available online

- High visibility within the field

- Retaining the copyright to your article

Submit your next manuscript at $\gg$ springeropen.com 